\title{
17. X-RAY MINERALOGY OF SEDIMENTS, DEEP SEA DRILLING PROJECT LEG $62^{1}$
}

\author{
Ulrich Nagel and Dieter Schumann, Institut für Sedimentforschung, \\ D-6900 Heidelberg, Federal Republic of Germany
}

\begin{abstract}
Sixty-nine sediment samples of Deep Sea Drilling Project Leg 62 were mineralogically examined by X-ray diffractometry, and gasometrically for their carbonate content. Most sediments were found to be carbonate-rich; some have up to $97 \% \mathrm{CaCO}_{3}$. Many chalk or limestone samples contain chert, accounting for high quartz percentages in some analyzed samples.
\end{abstract}

\section{INTRODUCTION}

During Leg 62, late Mesozoic and Cenozoic paleoenvironments in the central North Pacific Ocean were investigated. Four sites were drilled: Site 463 is in the western Mid-Pacific Mountains, Site 464 on northern Hess Rise, and Sites 465 and 466 on southern Hess Rise.

\section{METHODS}

Methods are essentially the same as those described in Mann and Müller (1980); they consist in measuring X-ray-diffraction peak heights or peak areas of individual minerals and relating them to percentages present. Carbonate contents have been determined by the "Karbonat-Bombe" method of Müller and Gastner (1971).

\section{RESULTS}

\section{Site 463 (Table 1; Fig. 1)}

Four sedimentary units have been identified, ranging in age from upper Barremian to Pleistocene.

Unit I, upper Albian to Pleistocene, was divided into two sub-units: Sub-unit IA is a pale-brown to white (sometimes mottled) Pleistocene to lower Eocene nannofossil ooze. Sub-unit IB consists of varicolored nannofossil and foraminifer chalk, ooze, and limestone of late Albian to late Maastrichtian age. Calcite is the main constituent, and the clay mineral suite is dominated by illite. Minor amounts of clinoptilolite have been found in samples of the $<2-\mu \mathrm{m}$ fractions of Sub-unit IB in Cores 7 to 10 . All cores of this sub-unit contain chert in varying amounts, and because this material is crystallized up to $8 \%$ quartz is present.

In Unit II, multicolored lower Aptian to middle Albian limestone, calcite is again the most important constituent. All analyzed samples are limestones or chalks and contain 8 to $10 \%$ quartz as reddish or gray pieces of chert, silicified foraminifers, or recrystallized radiolarians. The $<2-\mu \mathrm{m}$ fraction consists of smectite, plus variable amounts of illite.

In Unit III, tuffaceous and carbonaceous limestone, chert again occurs commonly as fragments and layers. Sample 26 (463-69-1, 145-146 cm), taken from an ash

\footnotetext{
${ }^{1}$ Initial Reports of the Deep Sea Drilling Project, Volume 62.
}

bed in Unit III, is rich in volcanic glass and smectite. Some feldspar and pyrite is present. Core 70 , from the lower part of Unit III, is characterized by olive-black to greenish-black layers that, according to shipboard analysis, are rich in organic carbon (see Site 463 report, this volume). Here we found opal-CT in addition to quartz, clinoptilolite, pyrite, and amorphous material. Clay mineral contents (smectite \pm illite) are variable.

Unit IV comprises interbedded pelagic and clastic limestones. Sample $31(463-82, \mathrm{CC}, 0-1 \mathrm{~cm})$ is a silicified limestone relatively rich in quartz and calcite. Sample 32 (463-84-2, 46-47 cm) is a clastic limestone with moderate quartz content and no clay minerals.

\section{Site 464 (Table 1; Fig. 2)}

At Site 464 , three sedimentary units were distinguished.

Unit I, late Miocene to Pleistocene, consists of clayey radiolarian ooze, clayey siliceous ooze with some calcareous nannofossils, and siliceous clay. Colors are different shades of brown and gray. This unit can be characterized mineralogically as containing moderate amounts of quartz and low amounts of feldspar, as well as carbonate-the latter decreasing from top to bottom. Illite dominates in the clay fraction; smectite, chlorite, and kaolinite constitute about $30 \%$ of the clay minerals.

Shipboard investigations have shown that Unit II is a brown clay which becomes phillipsite-rich in its lower parts. No zeolites were detected in our samples, the lowermost of which contains much quartz and some opal-CT. Goethite is a minor constituent. Reddishbrown chert chips from this part of Unit II were described by shipboard sedimentologists.

Chert is the dominant rock type recovered from Unit III. However, because our investigations were generally confined to samples from Units I and II, the data are incomplete.

\section{Site 465 (Table 1; Fig. 3)}

The lithologic subdivision of Site 465 is as follows: Unit I: light-gray and white nannofossil ooze and foraminifer nannofossil ooze to chalk (lower Cenomanian to Pleistocene), with chert fragments. Unit II: laminated limestone, nannofossil ooze and chert. Colors are 
Table 1. X-ray mineralogy of Holes 463, 464, 465, 465A, and 466.

\begin{tabular}{|c|c|c|c|c|c|c|c|c|c|c|c|c|c|c|c|c|c|}
\hline \multirow[b]{2}{*}{ Core } & \multirow[b]{2}{*}{ Section } & \multirow{2}{*}{$\begin{array}{c}\text { Interval } \\
\text { (cm) }\end{array}$} & \multirow[b]{2}{*}{ Sample } & \multirow{2}{*}{$\begin{array}{c}\text { Subb-bottom } \\
\text { Depth } \\
\text { (m) }\end{array}$} & & & & Bulk Mit & ralogy $(\%$ & of sedim & & & & Clay $\mathrm{N}$ & Mineralogy & $y$ (\% of clays) & \\
\hline & & & & & & Quartz & Feldspar & Carbonates & Opal-CT & Pyrite & Clinoptilolite & Phillipsite & Smectite & Illite & Chlorite & Kaolinite & Others \\
\hline Hole 4 & & & & & & & & & & & & & & & & & \\
\hline 1 & 3 & $20-22$ & 1 & 3.21 & 14.1 & 2.6 & - & 83.3 & - & - & - & - & $\overline{-}$ & 74.5 & 17,9 & 7.6 & - \\
\hline 4 & 4 & $82-84$ & 2 & 29.33 & 4.8 & 1.0 & - & 94.2 & - & - & - & - & 20 & 60.0 & & $20^{\mathrm{b}}$ & $\overline{c o t}$ \\
\hline 7 & 3 & $90-92$ & 3 & 47,41 & 5.1 & - & - & 94.9 & - & - & - & - & 17.1 & 77,7 & & $5.2^{\mathrm{b}}$ & Cpt \\
\hline 8 & 2 & $35-37$ & 4 & 54.86 & 7.7 & - & - & 92.3 & - & - & - & - & 19.5 & 73.4 & & $7.1^{\mathrm{b}}$ & $\mathrm{Cpt}$ \\
\hline 9 & 2 & $46-48$ & 5 & 64.47 & 5.8 & - & - & 94.2 & - & - & - & - & - & 100 & - & - & $\mathrm{Cpt}$ \\
\hline 10 & 4 & $\begin{array}{r}88-90 \\
46-58\end{array}$ & 6 & 77.39 & 14.4 & 1 & - & 84.6 & - & - & - & - & - & 100 & $\bar{z}$ & $z$ & cpt \\
\hline $\begin{array}{l}12 \\
17\end{array}$ & ${ }_{3}^{4}$ & $\begin{array}{l}56-58 \\
83-85\end{array}$ & $\frac{7}{8}$ & $\begin{array}{l}96.07 \\
9\end{array}$ & 6.4 & 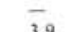 & - & $\begin{array}{l}93.6 \\
83.3\end{array}$ & - & - & $\overline{-}$ & $\overline{-}$ & $\overline{180}$ & 100 & - & $\overline{65}$ & Cpt \\
\hline 21 & 3 & $\begin{array}{c}83-85 \\
130-132\end{array}$ & $\begin{array}{l}8 \\
9\end{array}$ & $\begin{array}{l}142.34 \\
18081\end{array}$ & $\begin{array}{l}12.8 \\
11.5\end{array}$ & 3.9 & $=$ & $\begin{array}{l}83.3 \\
87.2\end{array}$ & $\bar{z}$ & $\overline{-}$ & $\bar{z}$ & $\bar{z}$ & $\begin{array}{l}18.9 \\
11.7\end{array}$ & $\begin{array}{l}74.5 \\
88.3\end{array}$ & $\bar{z}$ & 6.5 & $\bar{z}$ \\
\hline 22 & 3 & $110-112$ & 10 & $\begin{array}{l}190.11 \\
190.11\end{array}$ & 5.8 & ti. & $z$ & $\begin{array}{l}87.2 \\
94.2\end{array}$ & $\bar{z}+$ & $=$ & $=$ & $=$ & 22.6 & 77.4 & $=$ & $\bar{z}$ & $\bar{z}$ \\
\hline 24 & 3 & $61-63$ & i1 & 203.12 & 7.8 & - & - & 92.2 & - & - & - & - & 36.0 & 64.0 & - & - & - \\
\hline 26 & 3 & $124-126$ & 12 & 218.75 & 9.8 & 0.5 & - & 89.7 & - & - & - & - & 18.4 & 81.6 & - & - & - \\
\hline 30 & 1 & $106-108$ & 13 & 253.57 & 9.1 & 0.5 & - & 90.4 & - & - & - & - & 15.4 & 82.5 & 0.3 & 1.7 & - \\
\hline 34 & 3 & $70-72$ & 14 & 294.21 & 7.7 & - & - & 92.3 & - & - & - & - & 26.4 & 68.7 & & $4.8^{\mathrm{b}}$ & - \\
\hline 38 & 1 & $31-33$ & 15 & 328.82 & 7.9 & 1,1 & - & 91.0 & - & - & - & - & 2.9 & 87.0 & 1.4 & 8.7 & - \\
\hline 43 & 3 & $45-46$ & 16 & 379.45 & 5.3 & 0.5 & - & 94.2 & - & - & - & - & 6 & 83.7 & 5.2 & $\mathrm{a}^{\mathrm{b}} \mathrm{s.1}$ & - \\
\hline 48 & 3 & $50-52$ & 17 & 427.01 & 10.3 & 7.6 & - & 82.1 & - & - & - & - & 11.1 & 77.8 & & $11.1^{0}$ & - \\
\hline 50 & 1 & $36-38$ & 18 & $\begin{array}{l}433.37 \\
489.60\end{array}$ & 8.5 & 5.6 & - & 85.9 & - & - & - & - & 18.1 & 75.1 & 6.7 & $\bar{z}$ & $=$ \\
\hline $\begin{array}{l}55 \\
57\end{array}$ & 1 & $100-101$ & 19 & $\begin{array}{l}481.50 \\
-50\end{array}$ & $\begin{array}{r}9.7 \\
1.6\end{array}$ & 8.2 & - & $\begin{array}{l}82.1 \\
78.2\end{array}$ & $\bar{z}$ & - & - & $\overline{-}$ & $100^{7.7}$ & 92.3 & $=$ & $\overline{-}$ & $=$ \\
\hline $\begin{array}{l}57 \\
58\end{array}$ & ${ }_{3}^{1}$ & $\begin{array}{l}81-82 \\
7-8\end{array}$ & 20 & $\begin{array}{l}500.21 \\
512.07\end{array}$ & $\begin{array}{l}13.6 \\
17.8\end{array}$ & $\begin{array}{r}8.2 \\
10.4\end{array}$ & $\bar{z}+$ & $\begin{array}{l}78.2 \\
71.8\end{array}$ & $\bar{z}$ & $=$ & $=$ & $\bar{z}$ & $\begin{array}{l}100 \\
58.3\end{array}$ & $\overline{41.7}$ & $=$ & $=$ & $=$ \\
\hline 61 & i & $107-108$ & 22 & 536.57 & 16.8 & $\begin{array}{l}10.4 \\
7.8\end{array}$ & $=$ & $\begin{array}{l}17.8 \\
65.4\end{array}$ & $\bar{z}$ & $\bar{z}$ & $\overline{-}$ & $\Xi$ & 100 & - 1.7 & $\bar{z}$ & $z$ & $\overline{-}$ \\
\hline 62 & 2 & $15-20$ & 23 & 539.17 & 24.1 & 9.1 & 4.0 & 62.8 & - & - & - & - & 100 & - & - & - & - \\
\hline 66 & 2 & $84-86$ & 24 & 577.85 & 9.4 & 8.5 & - & 82.1 & - & - & - & - & 35.3 & 64.7 & - & - & - \\
\hline 67 & 2 & $108-109$ & 25 & 587.58 & 19.1 & 36.0 & - & 44.9 & - & - & - & - & 10 & 87.8 & 2.2 & - & - \\
\hline 69 & 1 & $145-146$ & 26 & 605.45 & 92.4 & - & 3.0 & - & - & 4.6 & - & - & 100 & - & - & - & - \\
\hline 70 & 1 & $96-97$ & 27 & 614.46 & 68.0 & 15.6 & - & 3.8 & 8 & 1 & 4 & - & 33.4 & 66.6 & - & - & - \\
\hline 70 & 6 & $40-41$ & 28 & 621.40 & 56.0 & 22.2 & - & 3.8 & 11 & 7 & $\overline{7}$ & - & $\overline{-}$ & - & - & - & - \\
\hline 70 & $?$ & $25-26$ & 29 & 622.75 & $\begin{array}{l}57.0 \\
50.8\end{array}$ & 3.9 & - & 32.0 & $=$ & - & 7 & - & 100 & $\overline{6} 6$. & - & - & - \\
\hline $\begin{array}{l}71 \\
82\end{array}$ & ${ }^{3}$ & $100-121$ & 30 & $\begin{array}{l}627.20 \\
7.20\end{array}$ & 52.8 & 5.2 & -1 & 41.0 & - & - & - & - & 83.5 & 16.5 & - & - & - \\
\hline $\begin{array}{l}82 \\
84\end{array}$ & $\underset{2}{\mathrm{CC}}$ & $\begin{array}{c}0-1 \\
46-47\end{array}$ & $\begin{array}{l}31 \\
32\end{array}$ & $\begin{array}{l}727.30 \\
738.96\end{array}$ & $\begin{array}{r}19.0 \\
6.6\end{array}$ & $\begin{array}{l}34.1 \\
12.6\end{array}$ & $=$ & $\begin{array}{l}26.9 \\
80.8\end{array}$ & $\bar{z}$ & $\bar{z}$ & $=$ & $\bar{z}$ & 100 & $\Xi$ & $\bar{z}$ & $z$ & $=$ \\
\hline Hole 4 & & & & & & & & & & & & & & & & & \\
\hline 2 & 3 & 91-93 & 1 & 7.42 & 69.4 & 10.4 & 2.0 & 19.2 & - & - & - & - & 11.1 & 67.4 & 15.0 & 6.5 & - \\
\hline 3 & 3 & $20-22$ & 2 & 16.21 & 66.2 & 7.2 & -1 & 25.6 & - & - & - & - & 12.4 & 72.4 & 4.2 & 11 & - \\
\hline 4 & 3 & $85-87$ & 3 & 26.36 & 84.4 & 13.0 & 2.6 & tr. & - & - & - & - & 9.0 & 70.0 & 10.5 & 10.5 & - \\
\hline $\begin{array}{l}5 \\
6\end{array}$ & 3 & $80-82$ & 4 & 35.81 & 87.6 & 10.4 & 2.0 & - & - & - & - & - & 8.9 & 68.8 & 12.7 & 9.6 & - \\
\hline $\begin{array}{l}6 \\
7\end{array}$ & 5 & $\begin{array}{l}94-96 \\
83-85\end{array}$ & $\begin{array}{l}5 \\
6\end{array}$ & $\begin{array}{l}48.45 \\
57.84\end{array}$ & $\begin{array}{l}91.2 \\
99.4\end{array}$ & 3.9 & 4.6 & - & $\bar{z}$ & $\bar{z}$ & $\bar{z}$ & $\bar{z}$ & $\begin{array}{l}89.0 \\
88\end{array}$ & 5.5 & & $5,5^{\circ}$ & $\bar{z}$ \\
\hline 9 & 1 & $\begin{array}{l}83-85 \\
75-77\end{array}$ & ${ }_{7}^{6}$ & $\begin{array}{l}5.84 \\
70.76\end{array}$ & $\begin{array}{l}93.4 \\
99.0\end{array}$ & $\begin{array}{l}2.6 \\
2.0\end{array}$ & 4.0 & $=$ & $=$ & $\overline{-}$ & $=$ & $\bar{z}$ & $\begin{array}{l}88 \\
13\end{array}$ & $\overline{67}$ & & $20^{\mathrm{b}}$ & $=$ \\
\hline 10 & 3 & $70-72$ & $8^{c}$ & 83.21 & 40.0 & 45.0 & $=$ & - & $\overline{5}$ & - & $=$ & $=$ & 13 & - & - & - & $\overline{-}$ \\
\hline Hole 4 & & & & & & & & & & & & & & & & & \\
\hline 3 & 3 & $108-110$ & 1 & 14.59 & 5.1 & - & - & 94.9 & - & - & tr. & - & 100 & - & - & - & $\mathrm{Cpt}$ \\
\hline $\begin{array}{r}4 \\
10\end{array}$ & $\begin{array}{l}1 \\
4\end{array}$ & $\begin{array}{l}49-50 \\
20-22\end{array}$ & ${ }_{3}^{2}$ & $\begin{array}{l}20.49 \\
81.71\end{array}$ & $\begin{array}{r}10.9 \\
7.7\end{array}$ & 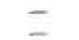 & $=$ & $\begin{array}{l}89.1 \\
92.3\end{array}$ & $\bar{z}$ & $\bar{z}$ & $\overline{t r}$ & $=$ & $=$ & $\overline{-}$ & $\bar{z}$ & $\bar{z}$ & $\overline{C p t}$ \\
\hline Hole 4 & $65 \mathrm{~A}$ & & & & & & & & & & & & & & & & \\
\hline 10 & 3 & $34-36$ & 1 & 127.85 & 2.6 & - & - & 97.4 & - & - & tr. & - & - & - & - & - & $\mathrm{Cpt}$ \\
\hline 12 & 2 & $5-7$ & 2 & 145.06 & 1.9 & - & - & 98.1 & - & - & tr. & - & - & - & - & - & $\mathrm{Cpt}$ \\
\hline 18 & 2 & $4-6$ & 3 & 202.05 & 10.3 & - & - & 89.7 & - & - & - & - & 100 & - & - & - & - \\
\hline 20 & 2 & $104-106$ & 4 & 222.05 & 3.9 & - & - & 96.1 & - & - & tr. & - & $\overline{0}$ & - & $\bar{z}$ & $=$ & Cpt \\
\hline 26 & 1 & 18-19 & 5 & 276.68 & 4.5 & - & - & 95.5 & - & - & - & - & 100 & - & - & - & 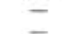 \\
\hline 27 & 2 & $24-25$ & 6 & 287.74 & 8.7 & - & -1.5 & 89.7 & - & $\overline{10}$ & - & $=$ & 100 & - & $\overline{-}$ & $\overline{-}$ & $\bar{z}$ \\
\hline 28 & 2 & $7-8$ & 7 & $\begin{array}{l}297.07 \\
306.36\end{array}$ & 27.0 & - & 6.4 & 66.6 & $\bar{z}$ & 1.0 & - & $\bar{z}$ & 100 & $\bar{z}$ & $=$ & $=$ & $\bar{z}$ \\
\hline $\begin{array}{l}29 \\
32\end{array}$ & 1 & $\begin{array}{c}136-137 \\
84-88\end{array}$ & ${ }_{9}^{8}$ & $\begin{array}{l}306.36 \\
334.36\end{array}$ & $\begin{array}{l}18.0 \\
20.5\end{array}$ & $=$ & $\bar{z}$ & $\begin{array}{l}82.0 \\
79.5\end{array}$ & $=$ & $\bar{z}$ & $\bar{z}$ & $=$ & $\overline{00}$ & $=$ & $=$ & $\bar{z}$ & $\bar{z}$ \\
\hline $\begin{array}{l}32 \\
33\end{array}$ & $\frac{1}{2}$ & $\begin{array}{l}84-88 \\
63-64\end{array}$ & 10 & $\begin{array}{l}334.36 \\
345.13\end{array}$ & $\begin{array}{l}20.5 \\
10.3\end{array}$ & $=$ & $=$ & $\begin{array}{l}79.5 \\
89.7\end{array}$ & $=$ & $\bar{z}$ & $=$ & $\bar{z}$ & $\begin{array}{l}100 \\
23,7\end{array}$ & $\overline{76.3}$ & 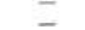 & $\bar{z}$ & $\bar{z}$ \\
\hline 34 & 1 & $\begin{array}{l}63-64 \\
80-81\end{array}$ & 11 & $\begin{array}{l}353.30 \\
353.30\end{array}$ & $\begin{array}{r}10.3 \\
3.2\end{array}$ & - & - & $\begin{array}{l}89.7 \\
96.8\end{array}$ & $\overline{-}$ & $\bar{z}$ & $\bar{z}$ & $\overline{-}$ & 42.5 & 57.5 & $=$ & $=$ & $=$ \\
\hline 36 & 2 & $112-113$ & 12 & 374.12 & 5.1 & - & - & 94.9 & - & - & - & - & 76.0 & 24 & - & - & - \\
\hline 37 & 1 & $29-30$ & 13 & 381.90 & 11.5 & - & - & 88 & - & - & - & - & 92.0 & 8.0 & - & - & - \\
\hline 38 & 2 & $84-85$ & 14 & 392. & 14 & - & - & 85.9 & - & - & - & - & 100 & - & - & - & - \\
\hline 39 & 2 & $36-37$ & 15 & & 11.5 & - & $\overline{-}$ & 88.5 & - & - & - & - & - & - & - & - & - \\
\hline 40 & 1 & $42-43$ & 16 & 409.92 & 88.8 & 1.6 & 3.0 & 3.8 & - & 2.8 & - & - & 100 & - & - & - & - \\
\hline Hole 4 & & & & & & & & & & & & & & & & & \\
\hline 1 & 1 & $57-59$ & 1 & 0.58 & 17.9 & 2.6 & & 79.5 & - & - & - & - & - & 85 & 10 & 5 & - \\
\hline 2 & 5 & $120-122$ & 2 & 15.21 & 20.1 & 4.6 & 1 & 74.3 & - & - & - & - & - & - & - & - & - \\
\hline 3 & 3 & $106-108$ & 3 & 21.57 & 11.5 & 1 & - & 88.5 & - & - & - & - & 18.5 & 59.3 & 13.1 & 9.1 & - \\
\hline 6 & 6 & $55-57$ & 4 & 54.06 & 16.0 & 3.3 & - & 84 & - & - & - & - & 6.0 & 65.0 & 16.0 & 13.0 & - \\
\hline 8 & 6 & $110-112$ & 5 & 73. & 19.4 & - & - & 75. & - & - & - & 5 & 24.5 & 64.5 & 7.0 & 4.0 & - \\
\hline 9 & 1 & & 6 & & 38.4 & - & - & 52.6 & - & - & - & 7 & 46.0 & 37.0 & 7.0 & 10.0 & - \\
\hline 15 & 3 & $20-22$ & 7 & 125.21 & 5.2 & - & - & 94.8 & - & - & - & - & 45.0 & 25.0 & 30.0 & & - \\
\hline 28 & $\mathrm{CC}$ & $20-21$ & 8 & $\begin{array}{r}255.00 \\
265.00\end{array}$ & 85.4 & 2.6 & - & $\bar{s}$ & 12.0 & - & - & - & 100 & - & - & - & - \\
\hline $\begin{array}{l}30 \\
34\end{array}$ & 1 & $\begin{array}{l}80-81 \\
8-87\end{array}$ & 9 & $\begin{array}{l}265.30 \\
293.86\end{array}$ & $\begin{array}{l}47.9 \\
208 .\end{array}$ & 13.0 & $\bar{z}$ & $\begin{array}{l}25.6 \\
78.2\end{array}$ & $\begin{array}{l}13.5 \\
1.0\end{array}$ & $\bar{z}$ & - & $\bar{z}$ & $\bar{z}$ & $\bar{z}$ & $\bar{z}$ & $\bar{z}$ & $\overline{-}$ \\
\hline & 1 & $86-87$ & 10 & 293.86 & 20.8 & & - & 78.2 & 1.0 & - & - & - & - & - & - & - & - \\
\hline
\end{tabular}

${ }_{\mathrm{b}}^{\mathrm{a}}$ Mainly volcanic glass.

Not separated, because of very small amounts.

Sample contains goethite.

shades of gray; chert is black in this unit. Unit III: trachyte.

Seven samples from Unit I were analyzed (Holes 465 and 465A). The main constituent is calcite. In addition, small quantities of clinoptilolite (bulk and $<2-\mu \mathrm{m}$ samples) were found. Only two samples yielded smectite.

In Unit II, high carbonate contents $(\sim 80-97 \%)$ were determined. Cores 33 to 37 yielded illite and smectite, whereas above and below this part of Unit II smectite is the only clay mineral. In sample 16 (465A-40-1, 42-43 $\mathrm{cm})$, taken from a carbon-rich limestone above volcanic basement, smectite (probably altered volcanic material) is the main component. In addition, we found $3 \%$ pyrite and feldspar, $16 \%$ quartz, and $\sim 4 \%$ calcite.

\section{Site 466 (Table 1; Fig. 4)}

The sedimentary sequence at this site comprises two major units. 
Unit I was divided into two sub-units: Sub-unit IA (upper Eocene to Pleistocene, white, gray, and brownish-white nannofossil ooze), and Sub-unit IB (mostly brownish-white late Turonian/Coniacian to mid-Eocene cherty nannofossil ooze). Calcite dominates the mineralogy of the analyzed samples. Minor admixtures include quartz and clay minerals.

Unit II (upper Albian to Turonian/Coniacian) is an olive-gray nannofossil chalk and limestone with some black chert. Sample $8(466-28, C C, 20-21 \mathrm{~cm})$, from the upper part of Unit II, is a black chert with some clay. It contains much amorphous material, and is relatively rich in opal-CT. Smectite also is present in this sample. The other samples of this unit are devoid of crystallized clay minerals.

Sample 9 (466-30-1, 80-81 cm), from a nannofossil chalk, also contains some chert (quartz, opal-CT, and amorphous components). Sample 10 (466-34-1, 86-87 $\mathrm{cm}$ ), having a similar lithology, is richer in carbonates; here, we found moderate amounts of amorphous material and minor opal-CT, in addition to calcite.

\section{ACKNOWLEDGMENTS}

This study was supported by the German Research Council (Deutsche Forschungsgemeinschaft). D. Godfrey typed and helped translate the manuscript. U. Kastner prepared the figures.

\section{REFERENCES}

Mann, U., and Müller, G., 1980. X-Ray mineralogy of Deep Sea Drilling Project Legs 51 through 53, western North Atlantic. In Donnelly, T., Francheteau, J., Bryan, W., Robinson, P., Flower, M., Salisbury, M., et al., Init. Repts. DSDP, 51, 52, 53, Pt. 1: Washington (U.S. Govt. Printing Office), 721-729.

Müller, G., and Gastner, M., 1971. The "Karbonat-Bombe," a simple device for the determination of the carbonate content in sediments, soils and other materials. N. Jahrb. Mineral. Mh., 10: 466-469. 

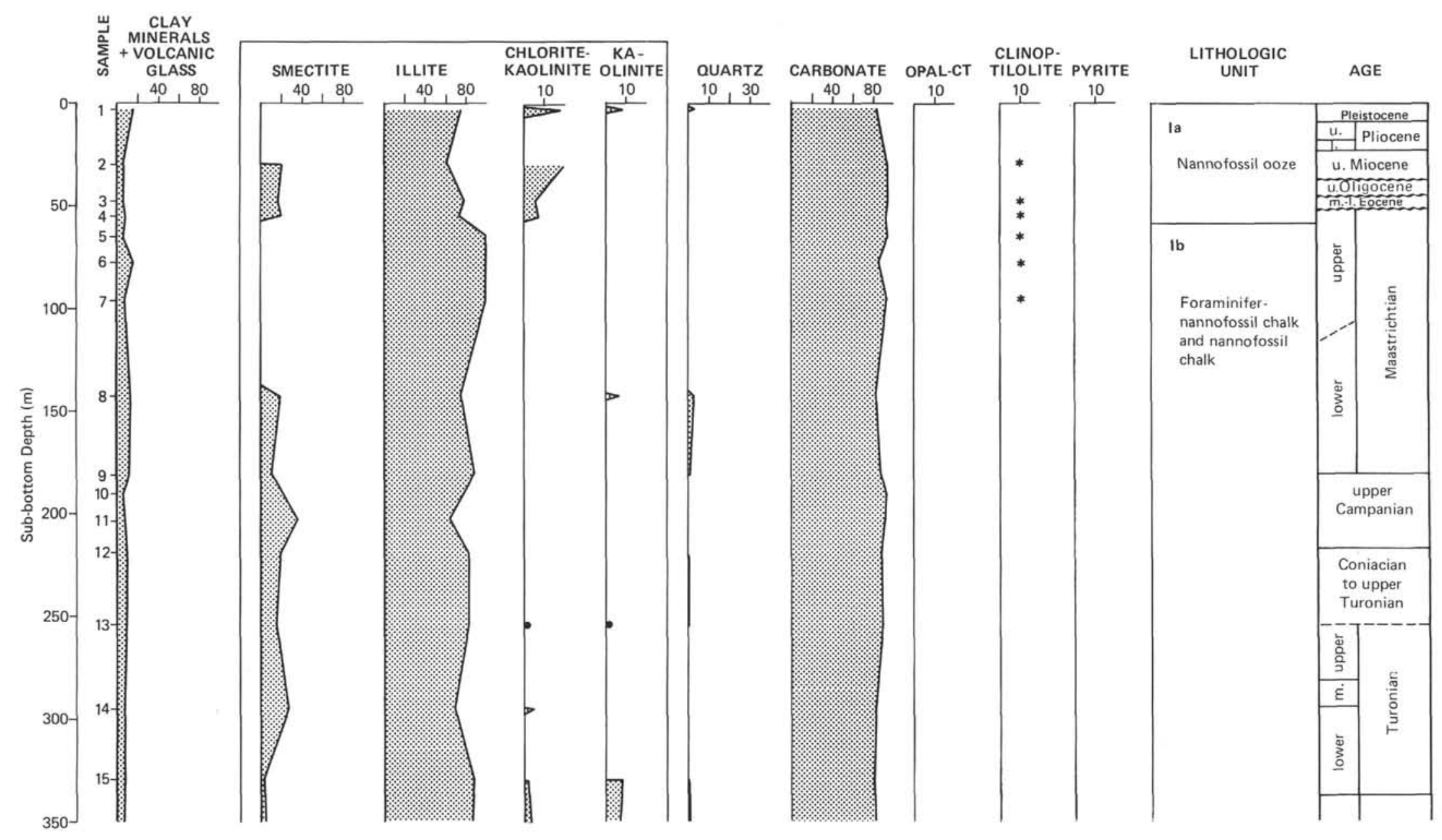

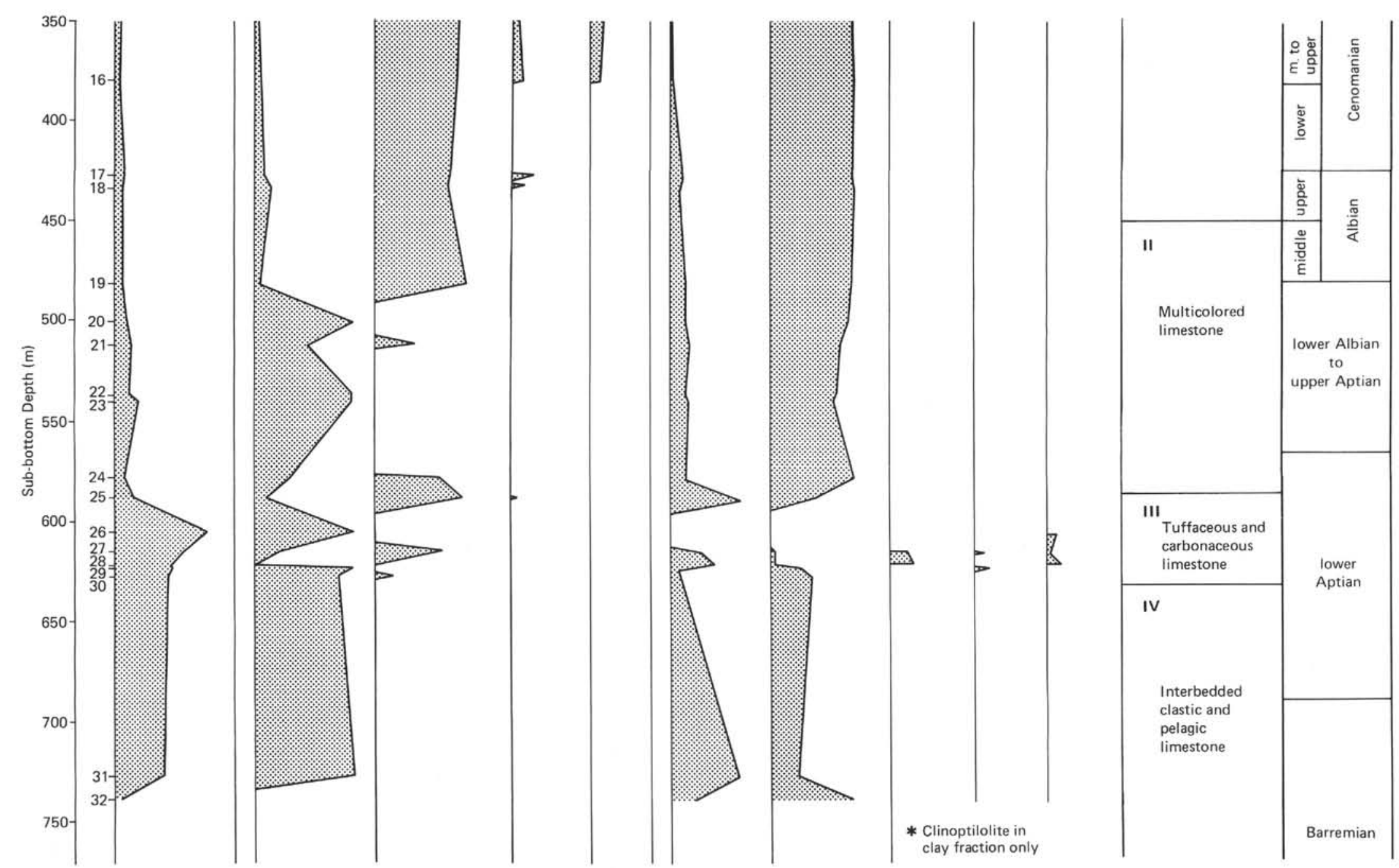

Figure 1. X-ray mineralogy of Hole 463. 


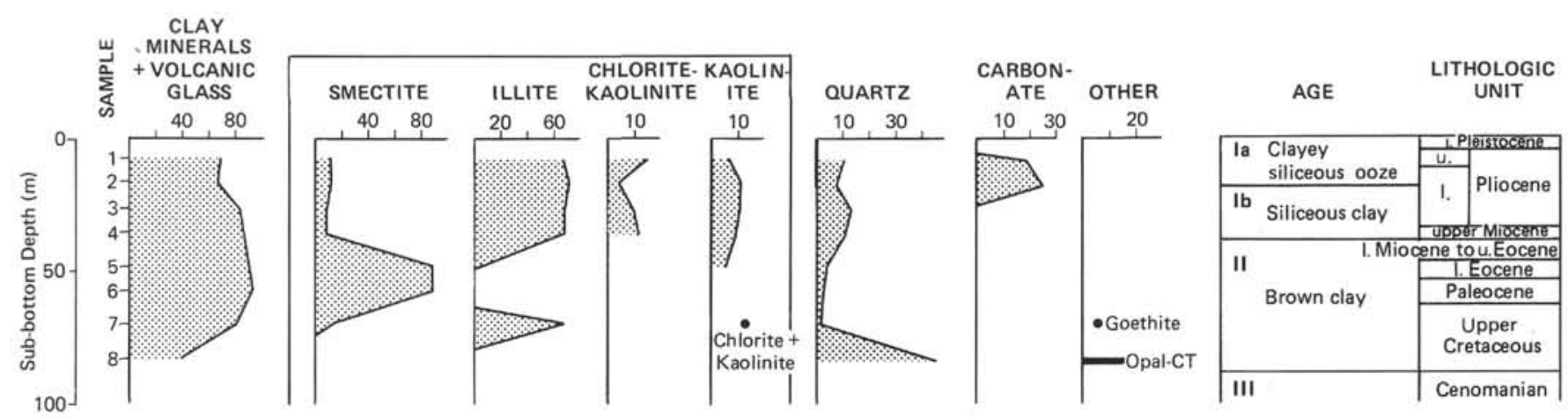

Figure 2. X-ray mineralogy of Hole 464.

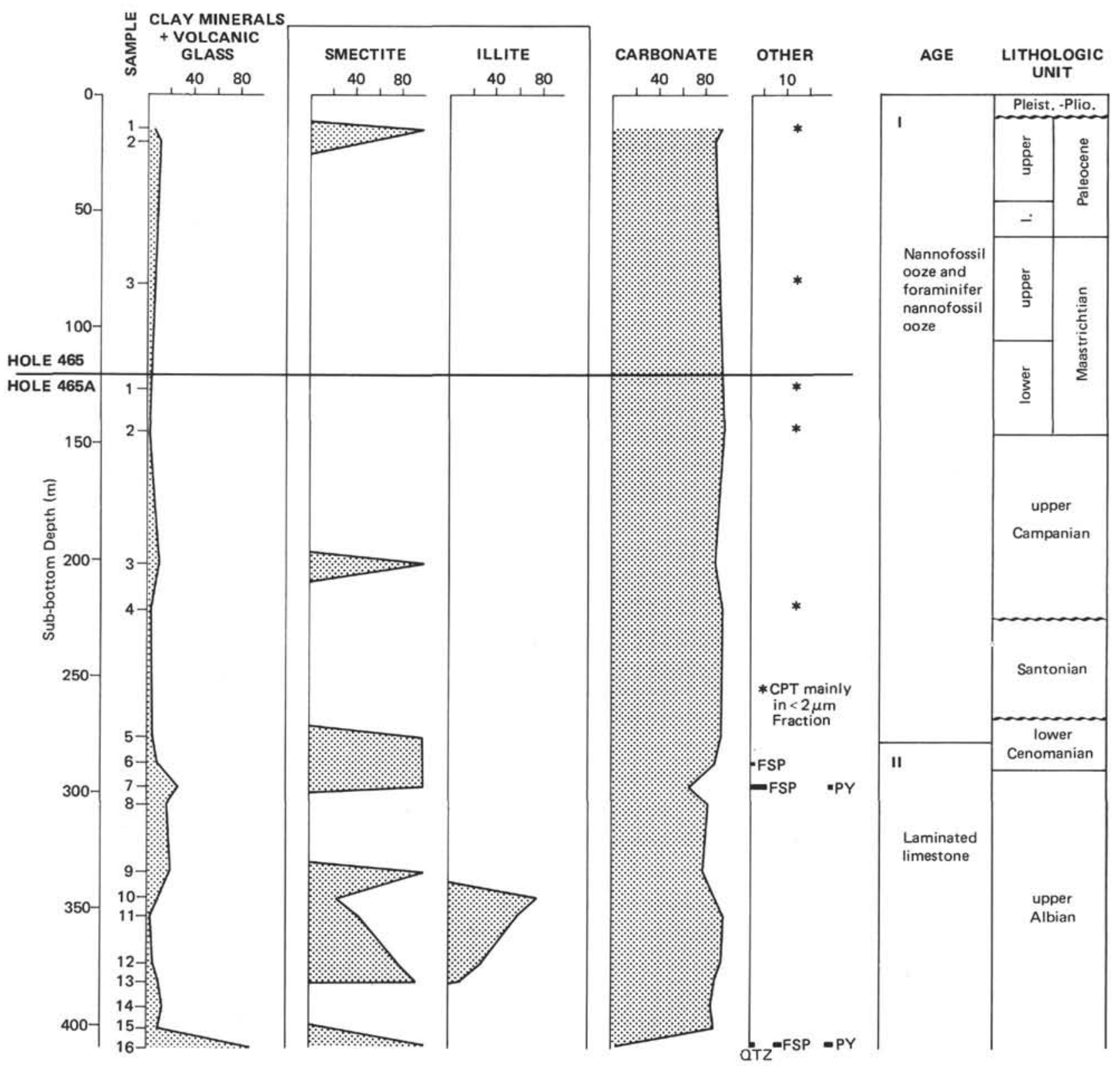

Figure 3. X-ray mineralogy of Holes 465 and 465A. 


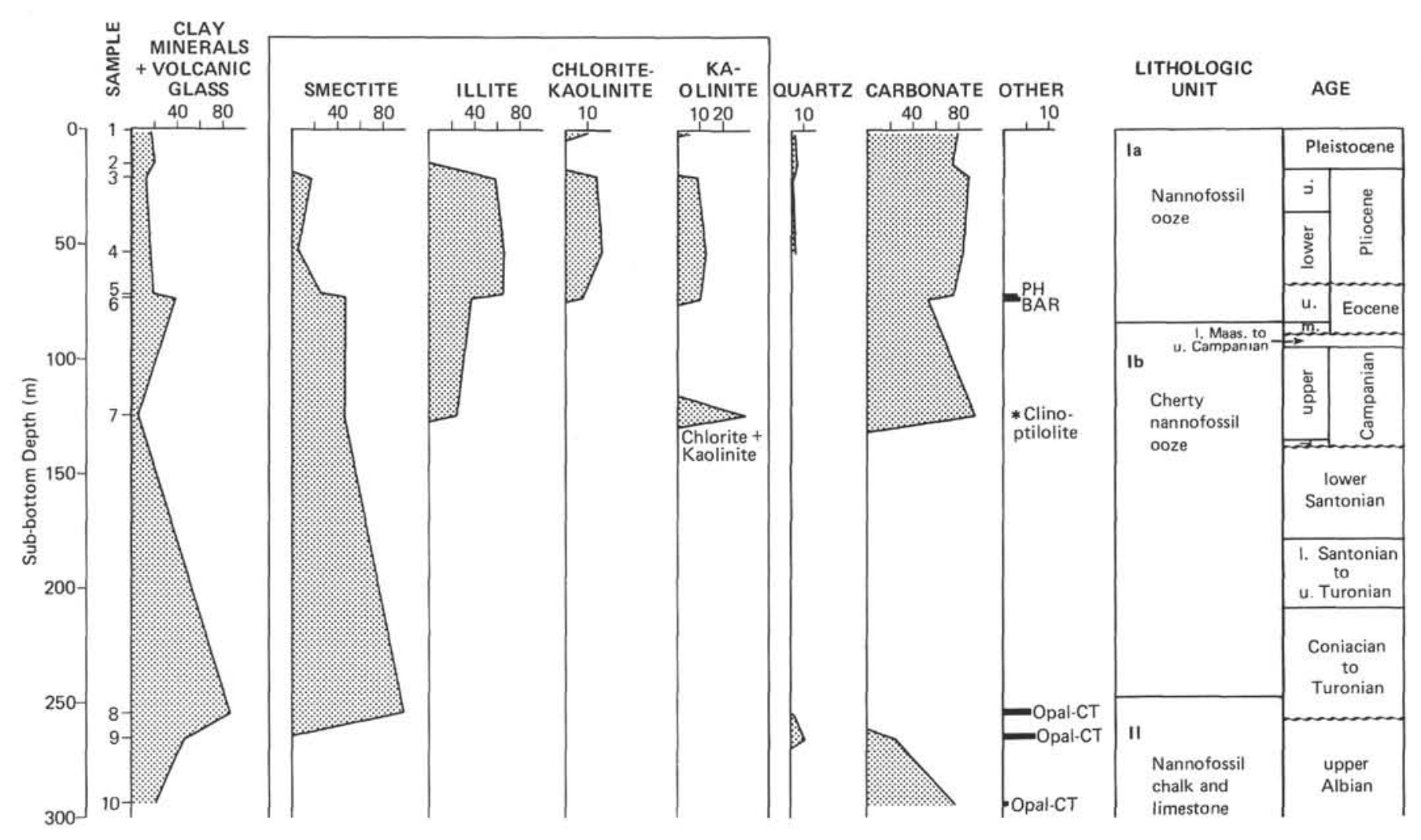

Figure 4. X-ray mineralogy of Hole 466. 